\title{
Daptomicina: características farmacológicas y aporte en el tratamiento de infecciones por cocáceas gram positivas
}

\author{
Rafael Araos, Patricia García, Leonardo Chanqueo y Jaime Labarca
}

\section{Daptomycin: pharmacological characteristics and its role in the treatment of gram positive infections}

Daptomycin recently made available in Chile, belongs to a new family of antimicrobials known as lypopeptides. Daptomycin has a unique mechanism of action and a potent bactericidal activity over susceptible agents. It is active against a number of clinically significant Gram positive cocci, including strains of Staphylococcus aureus and Enterococcus spp., both susceptible and resistant to classic antimicrobials. Daptomycin has been approved for clinical use in skin and soft tissue infections, and for $S$. aureus bacteremia in adult patients. Ongoing trials suggest that daptomycin is also useful in the treatment of other infections such as osteomyelitis, biofilm producing infections, and in immunocompromised patients, particularly onco-hematologic patients. The main adverse reaction associated with daptomycin use is myopathy, usually mild and reversible.

Key words: Daptomycin, Gram-positive infections, pharmacology, resistance.

Palabras clave: Daptomicina, infecciones por grampositivos, farmacología, resistencia.

\section{Introducción}

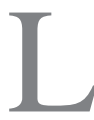
a importancia de las infecciones por cocáceas grampositivas ha aumentado en los últimos años, tanto aquellas de origen comunitario como las que se asocian a la atención de salud. Esta relevancia está dada en parte por un aumento en su incidencia, por el hecho que se asocian a una morbi-mortalidad considerable y porque determinan elevados costos económicos.

Muchas de las infecciones causadas por cocáceas grampositivas son producidas por microorganismos resistentes a los antibacterianos tradicionales, como es el caso de Staphylococcus aureus resistente a meticilina(SARM), Staphylococcus aureus resistente intermedio a vancomicina-(VISA, por las siglas en inglés "vancomycin intermediate Staphylococcus aureus”), Staphylococcus coagulasa negativa y Enterococcus spp. resistente a vancomicina (ERV), por lo que su tratamiento es un desafío para el médico clínico. Dentro de las nuevas alternativas terapéuticas con las que contamos para combatir infecciones por cocáceas grampositivas, daptomicina es el primer miembro de una nueva familia de antimicrobianos conocida como lipopéptidos, cuyo mecanismo de acción es único y confiere una gran potencia antimicrobiana. Esta revisión abarcará aspectos farmacológicos y clínicos de daptomicina ${ }^{1,2}$.

\section{Historia}

Daptomicina es un producto natural derivado del metabolismo de Streptomyces roseosporus y fue desarrollada en 1985 por la compañía Eli Lilly. Los estudios clínicos iniciales de seguridad, que utilizaron daptomicina en dosis fraccionada dos veces al día, mostraron una incidencia elevada de miopatía asociada al uso del fármaco, razón por la que se detuvo el proceso de desarrollo. Posteriormente, en 1997, y en respuesta a la emergencia de infecciones por cocáceas grampositivas difíciles de tratar, Cubist Pharmaceuticals retomó los estudios iniciales y demostró que la toxicidad muscular antes descrita se relacionaba con el fraccionamiento de la dosis y que, cuando daptomicina era utilizada una vez al día, era segura y efectiva. Este fue el punto de partida para estudios ulteriores que permitieron la aprobación de la molécula para su uso clínico, tanto en E.U.A. como en Europa (años 2003 y 2006, respectivamente) $)^{3-8}$.

\section{Estructura química y mecanismo de acción}

Daptomicina es el primer miembro de una nueva familia de antimicrobianos conocida como lipopéptidos cíclicos. Su estructura consiste en una molécula cíclica de 13 aminoácidos, que conforman un compuesto con un centro hidrofílico y un extremo hidrofóbico (Figura 1). Su fórmula química es C72H101N17026 y tiene un peso molecular de $1620.67^{2}$.

El mecanismo de acción postulado para daptomicina es único: en presencia de concentraciones fisiológicas de iones calcio (50 $\mu \mathrm{g} / \mathrm{ml})$, mediante el extremo hidrofóbico, las moléculas de daptomicina se insertan en la membrana citoplasmática bacteriana, donde se polimerizan y se disponen en la superficie formando canales iónicos. Estos canales permiten el transporte pasivo de potasio desde el medio intracelular al extracelular, alterando el potencial de membrana. Una vez ocurrido el cambio en el potencial, la célula es incapaz de seguir generando energía en forma de ATP, con la consiguiente detención de los
Facultad de Medicina Clínica Alemana/Universidad del Desarrollo, Santiago, Chile. Departamento de Medicina Interna (RA). Pontificia Universidad Católica de Chile.

Departamento de Laboratorios Clínicos (PG)

Departamento de Medicina Interna (JL)

Hospital San Juan de Dios de Santiago.

Laboratorio de Microbiología (LCh).

Los autores de esta revisión declaran no tener conflictos de interés y no recibieron compensación económica para la preparación de este artículo de revisión.

Recibido: 8 de diciembre de 2011 Aceptado:21 de febrero de 2011

Correspondencia a: Rafael Araos Bralic rafaaraos@gmail.com 
procesos vitales de la bacteria, lo que induce la muerte celular sin lisis. El hecho de tener actividad bactericida sin destrucción celular diferencia a daptomicina de otros antimicrobianos bactericidas y pudiera asociarse a un efecto positivo, modulando la respuesta inflamatoria asociada a la liberación de productos bacterianos que ocurre durante la lisis inducida por otros antimicrobianos ${ }^{1,2}$. Por último, en algunos reportes iniciales se mencionó que daptomicina pudiera además interferir en la síntesis de ácido lipoteicoico, pero estudios recientes han puesto en duda esta capacidad ${ }^{9}$.

\section{Farmacocinética}

La dosis aprobada de daptomicina en adultos es 4-6 $\mathrm{mg} / \mathrm{kg} /$ día por vía intravenosa. No obstante lo anterior, hay series de casos que reportan el uso de dosis altas de daptomicina (8-10 mg/kg/día) sin mayor toxicidad. Daptomicina tiene una farmacocinética lineal, no se acumula de manera significativa en voluntarios sanos y su vida media es 8-9 h. Alcanza concentraciones en estado de equilibrio luego de la tercera dosis. El volumen de distribución del fármaco es bajo $(0,1 \mathrm{~L} / \mathrm{kg})$, lo que implica que se concentra principalmente en el plasma y líquido intersticial. Tiene una alta unión a proteínas plasmáticas pero esta unión es reversible, por lo que la medición de la fracción libre de la molécula como índice de actividad puede subestimar su efecto real in vivo. Daptomicina no interactúa con el citocromo P450 y su eliminación es principalmente por vía renal (78\%). Debido a esto la dosis debe ajustarse cuando se administra en individuos con clearence de creatinina $<30 \mathrm{~mL} / \mathrm{min}$ (4-6 mg/kg cada 48 h). Daptomicina no cruza en forma efectiva la barrera hemato-encefálica y es inactivada por el surfactante pulmonar, razón por la cual no esté indicada en el tratamiento de neumonías ${ }^{2,10}$. Por último, se ha descrito que daptomicina tiene buena penetración a tejidos blandos, vegetaciones valvulares y en las bio-películas ${ }^{11}$.

(Nota del Editor: la farmacocinética de daptomicina en personas bajo 18 años de edad no ha sido cabalmente establecida. U.S. Food and Drug Administration. 3 de octubre 2011).

Figura 1. Daptomicina es un antimicrobiano lipopéptido cíclico derivado de la fermentación de Streptomyces roseosporus. Su nombre químico es: $\mathrm{N}$-decanoil-L-troptofil-D-asparaginil-L-aspartil-L-treonilglicilL-ornitil-L-aspartil-D-alanilL-aspartilglicil-D-seril-treo-3metil-L-glutamil-3-antraniloilL-alanina $э 1$-lactona.

\section{Farmacodinámica}

Daptomicina tiene actividad bactericida potente, rápida y concentración dependiente. Esta actividad la ejerce tanto sobre bacterias en fase activa de desarrollo como sobre aquellas que están en fase estacionaria. Además, daptomicina ha demostrado mantener su actividad contra cocáceas grampositivas resistentes a otros antimicrobianos como vancomicina, linezolid, quinupristina / dalfopristina y tigeciclina ${ }^{12}$. Se ha descrito un efecto post-antibiótico considerable, que varía según la especie bacteriana sobre la cual está actuando y puede ser hasta $6,8 \mathrm{~h}^{13}$. Los mejores parámetros farmacológicos para predecir la actividad in vivo son la relación entre área bajo la curva y la concentración inhibitoria mínima (ABC/CIM) en 24 h y la relación entre la concentración máxima y la CIM (Cmax/CIM). La probabilidad de alcanzar la relación óptima es mayor a 0,8 con la menor dosis recomendada, es decir, usando $4 \mathrm{mg} / \mathrm{kg} /$ día, en $80 \%$ de las veces se alcanzará la relación que predice la máxima actividad bactericida del fármaco ${ }^{2}$. Finalmente, se ha descrito sinergia in vitro entre daptomicina y otros antimicrobianos. En el caso de S. aureus, se ha observado sinergia entre daptomicina, rifampicina y algunos $\beta$-lactámicos; para el caso de Enterococcus spp., se ha reportado sinergia con rifampicina y ampicilina ${ }^{14}$.

\section{Espectro de acción}

Daptomicina es activa contra una gran variedad de cocáceas grampositivas, incluyendo todas las especies clínicamente relevantes de Staphylococcus, Streptococcus y Enterococcus. Además tiene acción in vitro contra algunos bacilos grampositivos, como Listeria spp., Corynebacterium spp., y contra algunas especies anaeróbicas. Daptomicina no es capaz de penetrar la membrana externa de agentes gramnegativos por lo que no es activa frente a éstos ${ }^{1,2}$. Es importante destacar que daptomicina no comparte mecanismo de acción alguno con otra molécula conocida; por lo tanto, especies resistentes como SARM, VISA y ERV se mantienen susceptibles a daptomicina. Como se ha comentado anteriormente, daptomicina tiene una excelente potencia bactericida, lo que se ve muy bien reflejado en el hecho que la gran mayoría de numerosas cepas estudiadas en E.U.A., Europa y en algunos países en Sudamérica han demostrado CIMs consistentemente bajas a daptomicina, incluyendo un elevado número de SARM, VISA y ERV ${ }^{15-17}$. En Chile, un estudio multicéntrico reciente, aún no publicado, encontró que la $\mathrm{CIM}_{50}$ y $\mathrm{CIM}_{90}$ para 338 cepas de SARM era 0,25 y 0,5 $\mu \mathrm{g} /$ $\mathrm{mL}$, respectivamente y para 164 cepas de ERV era $2 \mu \mathrm{g} /$ $\mathrm{mL}$ en ambos casos. Considerando los puntos de corte recomendados por entidades internacionales, 99,7\% de las cepas de SARM y 100\% de las cepas de ERV estuvieron en el rango de susceptibilidad ${ }^{31}$. 


\section{Experiencia clínica}

Las indicaciones para las cuales daptomicina ha sido aprobada en E.U.A. y Europa, en adultos*, son: tratamiento de infecciones complicadas de piel y tejidos blandos; tratamiento de bacteriemia y endocarditis derecha por $S$. aureus sensible a meticilina-(SASM) y SARM (Tabla 1). Actualmente existe gran experiencia en el uso de este fármaco para las indicaciones mencionadas y se han publicado numerosas series clínicas que reportan el uso de daptomicina en otras patologías infecciosas.

A continuación se describirán los ensayos clínicos fundamentales que permitieron la aprobación de daptomicina en adultos y se comentarán resultados de vigilancia post marketing relacionados al uso de daptomicina en las indicaciones clásicas y en otras situaciones clínicas seleccionadas.

\section{Infecciones complicadas y no complicadas de piel y tejidos blandos}

En el año 2004, Arbeit y cols., publicaron los resultados de dos ensayos clínicos de no inferioridad, que compararon daptomicina (4 mg/kg/día) versus penicilinas “penicilinasa-resistentes” (4-12 g/día) o vancomicina (1 g cada $12 \mathrm{~h}$ ), para el tratamiento de infecciones de piel y tejidos blandos. La mayoría de los pacientes incluidos en el estudio tenía infección del sitio quirúrgico, abscesos o úlceras infectadas. Se excluyeron infecciones leves y polimicrobianas. Se analizaron los resultados de 902 pacientes. La eficacia clínica de daptomicina fue 83,4\%, comparado con $84,2 \%$ del grupo comparador, cumpliendo criterios de no inferioridad. Tampoco hubo diferencias estadísticamente significativas cuando se analizaron subgrupos, como por ejemplo pacientes con pie diabético complicado (66\% éxito en el grupo de daptomicina versus $70 \%$ en el grupo comparador). Cabe destacar, que el grupo de daptomicina requirió significativamente menor tiempo de tratamiento comparado con el grupo de tratamiento estándar, lo que pudiera tener un impacto económico relevante ${ }^{5}$.

El análisis retrospectivo CORE (Cubicin ${ }^{\circledR}$ Outcomes Registry and Experience) y EU-CORE (E.U.A. y Europa, respectivamente) es un registro de fármaco-vigilancia financiado por la industria farmacéutica que busca comunicar datos relacionados al uso de daptomicina en la práctica diaria. En el reporte de CORE 2004 se incluyeron 522 pacientes con infecciones de piel y tejidos blandos, $64 \%$ con infecciones complicadas. El porcentaje de éxito clínico fue 97\% (considerando “cura” y “mejoría”) sin diferencias según tipo de infección ni según tipo de microorganismo asociado ${ }^{18}$. Así mismo, datos recientes de 484 pacientes con infección de piel y tejidos blandos provenientes del EU-CORE 2008-2009, mostraron una tasa de éxito clínico de $84 \%{ }^{19}$.

\section{Bacteriemia y endocarditis}

Fowler y cols., en el año 2006 comunicaron los resultados de un ensayo clínico de no inferioridad que comparó

Tabla 1. Indicaciones y dosis aprobadas para el uso de daptomicina en adultos

Indicación

Infecciones complicadas y no complicadas de piel y tejidos blandos

Bacteriemia y endocarditis infecciosa derecha por Staphylococcus aureus

daptomicina (6 mg/kg/día) contra vancomicina o una penicilina anti-estafilocóccica (dosis según indicación)ambas acompañadas inicialmente con una dosis baja de gentamicina- para el tratamiento de bacteriemia por $S$. aureus, con o sin endocarditis. El end point primario fue éxito clínico a las seis semanas después de haber completado el tratamiento. Se incluyeron pacientes con al menos un hemocultivo positivo para S. aureus. Al analizar un universo de 235 pacientes en total, tanto en el análisis por protocolo como por intención de tratar e intención de tratar modificada, daptomicina fue no inferior a sus comparadores. Los resultados fueron comparables entre los subgrupos de pacientes con bacteriemia complicada, endocarditis derecha e infección por SARM ${ }^{6}$.

A partir de los registros CORE y EU-CORE también se han obtenido datos acerca del uso de daptomicina en bacteriemia y endocarditis. Sakoulas presentó datos de 126 pacientes con bacteriemia, en su mayoría por SARM, ERV y Staphylococcus coagulasa negativa. En general, daptomicina no fue el antimicrobiano de primera línea y el porcentaje de éxito clínico fue $89 \%{ }^{20}$. Levine reportó los resultados de 49 pacientes con endocarditis infecciosa, en su mayoría con compromiso izquierdo. Staphylococcus aureus resistente a meticilina y ERV fueron los agentes más frecuentemente aislados, la dosis utilizada fue siempre mayor a $6 \mathrm{mg} / \mathrm{kg} /$ día y el porcentaje de éxito clínico fue $63 \%{ }^{21}$. Por último, datos recientes obtenidos del EU-CORE mostraron el resultado de 308 pacientes tratados por bacteriemia en distintos centro europeos entre los años 2008 y 2009. La mitad de los casos se relacionó a dispositivos intravasculares y los agentes más frecuentemente aislados fueron $S$. coagulasa negativa, $S$. aureus (con una proporción significativa de SARM) y finalmente Enterococcus spp. Para los pacientes con bacteriemia relacionada a catéter el porcentaje de éxito terapéutico fue $76 \%$ y para aquellos con bacteriemia no relacionada a catéter, el éxito fue $72 \%{ }^{22}$.

\section{Otras situaciones clínicas}

En datos del registro CORE publicados en el año 2007, se describen los resultados del tratamiento con daptomicina de 148 casos de pacientes con osteomielitis. Diez y seis pacientes tuvieron bacteriemia asociada y 17 pacientes tenían alojado material protésico. En el grupo que logró seguirse después del alta (67 pacientes), el porcentaje de éxito fue $82 \%$. La cirugía concomitante y el uso de dosis $>6 \mathrm{mg} / \mathrm{kg} /$ día se asociaron a un menor riesgo de fracaso ${ }^{23}$. Por otra parte, ya se ha comentado la capacidad de daptomicina para actuar en forma efectiva, al 
menos in vitro, sobre infecciones asociadas a bio-película, como es el caso de infección de prótesis ortopédicas y de dispositivos intravasculares. A la fecha existen algunos estudios en modelos animales que confirman estos hallazgos ${ }^{24}$. Esta propiedad convierte a daptomicina en una alterativa interesante para investigar su utilidad clínica en este tipo de infecciones difíciles de tratar. Por último, es importante destacar que existe evidencia creciente a favor del uso de daptomicina en unidades onco-hematológicas, incluyendo pacientes con neutropenia febril ${ }^{25}$.

\section{Infecciones por Enterococcus spp}

Se ha descrito en revisiones recientes que daptomicina mantiene su actividad bactericida concentración dependiente sobre cepas de enterococo, incluyendo cepas de E. faecium resistente a vancomicina. Debido al hallazgo de CIMs relativamente altas para Enterococcus spp. en relación a lo descrito para cepas de Staphylococcus spp y a reportes de fracaso terapéutico al usar daptomicina como antimicrobiano único en dosis estándar $(6 \mathrm{mg} / \mathrm{kg} /$ día), parece razonable que en los casos de infecciones graves por E. faecium, en los cuales se decida indicar daptomicina, se utilicen dosis altas (> $8 \mathrm{mg} / \mathrm{kg} /$ día) y se combine junto a otros antimicrobianos como ampicilina, rifampicina, gentamicina y tigeciclina ${ }^{26}$.

(Nota del Editor: la indicación de daptomicina en personas bajo 18 años de edad no ha sido establecida aún. U.S. Food and Drug Administration. 3 de octubre 2011).

\section{Resistencia}

Al día de hoy, la resistencia a daptomicina es un hecho muy poco frecuente, aunque se han descrito algunas cepas de $S$. aureus resistentes y el mecanismo sugerido se ha relacionado a la acumulación de mutaciones puntuales de genes que codifican productos implicados en distintas funciones del metabolismo bacteriano. Otras alteraciones mencionadas se asocian a alteraciones en la unión de daptomicina a la membrana celular ${ }^{27}$. En el caso de Enterococcus spp., la resistencia es también un fenómeno poco común; sin embargo, las CIMs para daptomicina tienden a ser más elevadas que lo encontrado en Staphylococcus spp., y por otra parte, existe evidencia que advierte acerca del posible desarrollo de resistencia intra-tratamiento con daptomicina ${ }^{28}$. Estos hallazgos, sugieren que se debe implementar una vigilancia de la susceptibilidad a daptomicina de cepas de cocáceas grampositivas.

\section{Toxicidad}

La principal toxicidad asociada al uso de daptomicina es muscular, pudiendo ir desde una elevación asintomática de los niveles séricos de creatin-fosfoquinasa (CPK) hasta miopatía clínicamente relevante. La alteración de CPK es reversible, en la mayoría de los casos, con la suspensión del medicamento. Como ya se ha mencionado, el uso de daptomicina una vez al día disminuyó significativamente la incidencia de este efecto adverso. La incidencia estimada para elevación asintomática de CPK varía entre 3 y 7\% y para miopatía grave $\sim 0,2 \% \%^{5,7}$. Si bien el uso de dosis altas de daptomicina (10-12 mg/kg/día) en voluntarios sanos y series de casos no han observado mayor incidencia de alteraciones musculares, un estudio encontró una relación directa entre la concentración plasmática mínima y el riesgo de miopatía, lo que sugiere que la dosis máxima diaria pudiera tener relación con el riesgo de miopatía ${ }^{29}$. En este mismo estudio, la toxicidad muscular también se relacionó en forma directa con el tiempo de uso del fármaco. Dado el riesgo potencial de daño muscular asociado al uso de daptomicina, deben medirse periódicamente niveles de $\mathrm{CPK}$, especialmente en aquellos pacientes con un riesgo basal aumentado (insuficiencia renal, CPK basal elevada y uso concomitante de estatinas, fibratos y ciclosporina). Daptomicina debe suspenderse cuando hay mialgias o elevación de CPK > 5 veces el valor normal.

Efectos adversos infrecuentes asociados al uso de daptomicina incluyen casos aislados de alteraciones neurológicas (parestesias, disestesias) y de neumonía eosinofílica ${ }^{30}$.

\section{Conclusión}

Daptomicina es un nuevo antibacteriano con excelente actividad contra infecciones por cocáceas grampositivas, incluyendo cepas resistentes a los antimicrobianos de prescripción habitual. Las indicaciones en adultos para las cuales daptomicina ha sido aprobada son: infecciones de piel y tejidos blandos y bacteriemia y endocarditis derecha por $S$. aureus, tanto susceptible como resistente a meticilina. Su perfil de escasos efectos adversos lo convierte en un medicamento seguro y que puede ser monitorizado. Esperamos en un futuro próximo poder disponer de datos de estudios clínicos de buena calidad acerca del comportamiento de daptomicina en infecciones causadas por Enterococcus spp y aquellas relacionadas con la producción de bio-película, como las asociadas a prótesis ósteo-articulares.

\section{Resumen}

Daptomicina es un anti-infeccioso de reciente introducción en Chile, miembro exclusivo de una nueva familia de antimicrobianos conocida como lipopéptidos cíclicos. Tiene un mecanismo de acción único que le confiere un potente efecto bactericida sobre los microorganismos susceptibles. Su especto antimicrobiano comprende cocáceas grampositivas de importancia clínica como 
Staphylococcus aureus y Enterococcus spp., incluyendo cepas resistentes a antimicrobianos habituales. Está aprobada para el uso clínico en infecciones de piel y tejidos blandos y bacteriemia complicada y no complicada por $S$. aureus, en adultos. Estudios en curso sugieren que será una alternativa útil en otras infecciones frecuentes como osteomielitis, infecciones asociadas a dispositivos ortopédicos, infecciones asociadas a biopelículas e infecciones en hospederos inmunosuprimidos, en particular en pacientes onco-hematológicos. El principal efecto adverso asociado al uso de daptomicina es la toxicidad muscular, observándose miopatía reversible, la mayoría de las veces asintomática, en aproximadamente $3 \%$ de los pacientes que utilizan el fármaco.

\section{Referencias}

1.- Alder J. Daptomycin, a new drug class for the treatment of Gram-positive infections. Drugs Today 2005; 41 (2): 81-90.

2.- Kosmidis C, Levine D. Daptomycin: pharmacology and clinical use. Expert Opin Pharmacother 2010; 11 (4): 615-25.

3.- Livermore D. Future directions with daptomycin. J Antimicrob Chemother 2008; 62 (S3): iii41-iii49.

4.- Eliopoulos G, Willey S, Reiszner E, Spitzer P G, Caputo G, Moellering R C Jr. In vitro and in vivo activity of LY 146032, a new cyclic lipopeptide antibiotic. Antimicrob Agents Chemother 1986; 30: 532-5.

5.- Arbeit R D, Maki D, Tally F P, Campanaro E, Eisenstein B I. The safety and efficacy of daptomycin in the treatment of complicated skin and skin structure infections. Clin Infect Dis 2004; 38: 1673-81.

6.- Fowler V G Jr, Boucher H W, Corey G R, Abrutyn E, Karchmer A W, Rupp M E, et al. Daptomycin versus standard therapy for bacteremia and endocarditis caused by Staphylococcus aureus. N Engl J Med 2006; 355 (7): 653-65.

7.- Oleson F B Jr, Berman C L, Kirkpatrick J B, Regan K S, Lai J J, Tally F P. Once-daily doping in dogs optimizes daptomycin safety. Antimicrob Agents Chemother 2000; 44: 294853.

8.- Eisenstein B, Oleson F Jr, Baltz R. Daptomycin: from the mountain to the clinic, with essentials help from Francis Tally, MD. Clin Infect Dis 2010; 50: S10-5.

9.- $\quad$ Laganas V, Alder J, Silverman J. In vitro bactericidal activities of daptomycin against Staphylococcus aureus and Enterococcus faecalis are not mediated by inhibition of lipoteichoic acid biosynthesis. Antimicrob Agents Chemother 2003; 47(8): 2682-4

10.- Hawkey P. Pre-clinical experience with daptomycin. J Antimicrobial Chemother 2008; 62(S3): iii7-iii14.

11.- Raad I, Hanna H, Jiang Y, Dvorak T, Reitzel R, Chaiban G, et al. Comparative activities of daptomycin, linezolid, and tigecycline against catheter-related methicillin-resistant Staphylococcus bacteremic isolates embedded in biofilm. Antimicrob Agents Chemother 2007; 1656-60.

12.- Anastasiou D M, Thorne G M, Luperechio S A,
Alder J D. In vitro activity of daptomycin against clinical isolates with reduced susceptibilities to linezolid and quinupristin/ dalfopristin. Int J Antimicrob Agents 2006; 28: 385-8.

13.- Hanberger $H$, Nilsson, Maller R, Isaksson B. Pharmacodynamics of daptomycin and vancomycin on Enterococcus faecalis and Staphylococcus aureus demonstrated by studies of initial killing and postantibiotic effect and influence of $\mathrm{Ca}+$ and albumin on these drugs. Antimicrob Agents Chemother 1991; 35 (9): 1710-6.

14.- Steenbergen J, Mohr J, Thorne G. Effects of daptomycin in combination with other antimicrobial agents: a review of in vitro and animal model studies. J Antimicrobial Chemother 2009; 64: 1130-8.

15.- Sader H, Jones R. Evaluation of daptomycin activity tested against 35058 bacterial strains from hospitalized patients: Summary of a 7 year surveillance program for North America (20022008). $47^{\text {th }}$ Annual Meeting of the Infectious Diseases Society of America; Philadelphia, PA. October 29- November 1. Abstract 199.

16.- Sader H S, Watters A A, Fritsche T R, Jones R N. Daptomycin antimicrobial activity tested against methicilin-resistant staphylococci and vancomycin-resistant enterococci isolated in European medical centres (2005). BMC Infect Dis 2007; 7: 29.

17.- Gales A, Sader H, Ribeiro J, Zoccoli C, Barth A, Pignatari A. Antimicrobial susceptibility of Gram-positive bacteria isolated in Brazilian hospitals participating in the SENTRY program (2005-2008). Braz J Infect Dis 2009; 13 (2): 90-8.

18.- Seaton R. Daptomycin: rationale and role in the management of skin and soft tissue infections. J Antimicrobial Chemother 2008; 62(S3): iii15iii23.

19.- Cogo A, Dailiana Z, Gargalianos-Kakolyris P, Quast T, Prisco V, Segado A, et al. Complicated skin and soft tissue infections (cSSTIs) treated with daptomycin in the European Cubicin ${ }^{\circledR}$ Outcomes Registry and Experience (EUCORE $\left.^{\text {sm }}\right) .20^{\text {th }}$ European Congress of Clinical Microbiology and Infectious Diseases; Vienna, Austria. 10-13 April 2010. Poster 1227.

20.- Sakoulas G, Golan Y, Lamp K C, Friedrich L V, Russo R, et al. Daptomycin in the treatment of bacteremia. Am J Med 2007; 120: S21-7.

21.- Levine D, Lamp K. Daptomycin in the treatment of patients with infective endocarditis: experience from a registry. Am J Med 2007; 120: S28-33.

22.- Galloway A, Galván-Guijo B, González-Ruiz A, Rodríguez A, Almirante B, Lejko-Zupanc T, et al. Daptomycin (DAP) in the treatment of bacteremia: clinical experience in Europe. $20^{\text {th }}$ European Congress of Clinical Microbiology and Infectious Diseases; Vienna, Austria. Poster 1228.

23.- Lamp K, Friederich L, Mendez-Vigo L, Russo R. Clinical experience with daptomycin for the treatment of patients with osteomyelitis. Am J Med 2007; 120: S13-20.

24.- John A K, Baldoni D, Haschke M, Rentsch K, Schaerli P, Zimmerli W, et al. Efficacy of daptomycin in implant-associated infection due to methicillin-resistant Staphylococcus aureus: importance of combination with rifampin. Antimicrob Agents Chemother 2009; 53 (7): 2719-24.

25.- Rolston K, McConnell S, Brown J, Lamp K. Daptomycin use in patients with cancer and neutropenia: data from a retrospective registry. Clin Adv Hematol \& Oncol 2010; 8 (4): 249-57.

26.- Arias C A, Contreras G A, Murray B E. Management of multidrug-resistant enterococcal infections. Clin Microbiol Infect 2010; 16: $555-62$.

27.- Kaatz G, Lundstrom T, Seo M. Mechanisms of daptomycin resistance in Staphylococcus aureus. Int J Antimicrob Agents 2006; 28 (4): 280-7.

28.- Arias C A, Panesso D, McGrath D M, Qin X, Mojica M F, Miller C, et al. Genetic basis for in vivo daptomycin resistance in enterococci. $\mathrm{N}$ Engl J Med 2011; 365 (10): 892-900.

29.- Bhavnani S, Rubino C, Ambrose P, Drusano L. Daptomycin exposure and the probability of elevations in the creatinine phosphokinase level: data from a randomized trial of patients. Clin Infect Dis 2010; 50 (12): 1568-74.

30.- Lal Y, Assimacopoulos P. Two cases of daptomycin-induced eosinophilic pneumonia and chronic pneumonitis. Clin Infect Dis 2010; 50: 737-40.

31.- García P, Vecchiola M, Juliet C, Chanqueo L, Cifuentes M, Silva F, et al. Actividad antimicrobiana de daptomicina: primer estudio en cocáceas Gram positivas resistentes en Chile. XXVIII Congreso Chileno de Infectología, Coquimbo, 30 de Noviembre-1 y 2 de diciembre 2011. Pág S65 PO 11. 